\title{
Risk factors for stroke and neurocognitive disorders: the role of pre- and post-stroke physical activity
}

\author{
Bogdan Constantin Monac, Roxana Chiriță, \\ Dan Iulian Cuciureanu, Georgiana Mihaela Monac
}

\begin{abstract}
Bogdan Constantin Monac - M.D., PhD Student, Neurology fellow, Neurology department, University of Medicine and Pharmacy „Grigore T. Popa” Iași, Romania

Roxana Chiriță - M.D., PhD, Professor, University of Medicine and Pharmacy "Grigore T. Popa" Iași, Senior psychiatrist Socola Institute of Psychiatry, Iași, Romania

Dan Iulian Cuciureanu - M.D., PhD, Neurology department, University of Medicine and Pharmacy „Grigore T. Popa” Iași, Senior neurologist ”Nicolae Oblu” Emergency Clinical Hospital, 1st Neurological Clinic, Iasi, Romania

Georgiana Mihaela Monac - M.D., Gastroenterology fellow, University of Medicine and Pharmacy „Grigore T. Popa” Iași, Romania
\end{abstract}

\begin{abstract}
Stroke is one of the main problems for health systems, being a major cause of mortality and the second leading cause of DALYs. Through the development of medicine in recent years, mortality caused by cerebral vascular events and physical dysfunction tend to decrease. This turns mental dysfunction into the main cause of concern for the health systems and implicitly for society. Global cognitive decline after stroke is common, with a prevalence of approximately $44 \%$ in the first 6 months after the acute event. This has an effect on functional recovery abilities and increases the risk of developing major neurocognitive disorder. The long-term mortality is two to six times higher in patients with post-stroke dementia. Ten potentially modifiable risk factors are collectively associated with approximately $90 \%$ of the risks that can be attributable to the population for stroke. Physical activity decreased population attributable risk by about $53 \%$ in all types of stroke. Physical activity is associated with a decreased risk of stroke and mortality, as well as favorable results after stroke, which makes it a potential preventive measure against cognitive decline. An increased level of physical activity performed prior to stroke is independently associated with higher cognitive performance after stroke. The combined use of several measures with a vascular protective role, applied since middle age, could prevent several years of cognitive aging.
\end{abstract}




\section{Stroke, neurocognitive disorder, physical activity.}

\section{Stroke and neurocognitive disorders}

Stroke is one of the main problems for health systems around the world, being a major cause of mortality and disability. According to the 2019 Global Burden Disease, Injuries and Risk Factors Study (GBD), stroke is the second leading cause of DALYs (Disabilityadjusted life years) for both age group 50-74 years and for people over 75 years. (1)

The traditional definition of stroke is based on the sudden loss of neurological function due to infarction or hemorrhage in a relevant part of the brain, retina or spinal cord. According to an updated definition, stroke is an acute episode of focal dysfunction of the brain, retina or spinal cord that lasts more than 24 hours, or of any duration if there is an imaging lesion (CT, MRI) or at autopsy, which can justify the symptoms. (2)

The main symptoms of a stroke, regardless of etiology, are muscular weakness, expressive or receptive aphasia and cognitive dysfunction. Motor deficit along with language disorders are symptoms that alert the patient from the first moments of the vascular event and implicitly have the greatest psychological impact. Cognitive dysfunction does not have the same clinical resonance, it can have a long and silent evolution, which is why it alerts the patient or his entourage in late stages.

Through the development of medicine in recent years, both in terms of diagnosis with high-fidelity paraclinical explorations, and through topical treatments that allow rapid and successful interventions, mortality caused by cerebral vascular events and physical dysfunction tend to decrease. This turns mental dysfunction into the main cause of concern for the health system and implicitly for society.

Cognition encompasses many aspects of intellectual functions and processes such as attention, knowledge development, memory and working memory, judgment and evaluation, reasoning and "calculation", problem solving and decision making, understanding and language production. Cognitive processes use existing knowledge and generate new knowledge.

According to the fifth edition of the Diagnostic and Statistical Manual of Mental Disorders abbreviated as DSM-5, published by the American Psychiatric Society in 2013, neurocognitive disorder is divided into minor neurocognitive disorder, major neurocognitive disorder and delirium. Minor neurocognitive disorder is manifested by a modest cognitive decline (compared to a previous level of performance), reported either by the patient, entourage, treating physician or by standardized neuropsychological testing, or, in their absence, by other clinical evaluations that can be quantified. This syndrome is characterized by impairment of one or more cognitive domains, such as complex attention, executive function, memory and learning, language, perceptual-motor function, or social cognition. Cognitive decline does not interfere with independence from complex daily instrumental activities, but requires greater effort, compensatory strategies, or accommodation in the conduct of daily activities, such as paying bills or administering medication. Also, this cognitive impairment does not occur exclusively in the context of delirium and is not explained by 
s

another mental disorder, for example schizophrenia. (3)

Major neurocognitive disorder, corresponding to dementia, is manifested by a substantial impairment of one or (usually) several cognitive areas. The dysfunction must be sufficiently significant to interfere with independence from daily activities (excluding motor / sensory sequelae of a cerebral vascular event). (3)

Over time, there has been a significant evolution of the terminology that defines the cognitive syndrome associated with cerebrovascular risk factors and their manifestations, especially in terms of describing dementia. About 40 years ago, the term "multi-infarct dementia" (MID) was used to identify patients who developed dementia after multiple strokes. Subsequently, the expression "vascular dementia" (VaD vascular dementia) was used, regardless of the pathogenesis of the vascular lesion, ischemic or hemorrhagic, single or multiple infarction.

Global cognitive decline after stroke is common, with a prevalence of approximately $44 \%$ in the first 6 months after the acute event. (5) This has an effect on functional recovery abilities and increases the risk of developing major neurocognitive disorder.

Vascular cognitive impairment (VCI) are a heterogeneous group of neurocognitive disorders, the essential feature being that cognitive decline is mainly attributed to cerebrovascular disease. This group includes both vascular dementia and cognitive impairment that do not meet the criteria for dementia. $(6,7)$

The most severe form of cognitive impairment by vascular origin is vascular dementia. Dementia is not always a direct consequence of cerebrovascular lesions, it can be degenerative, with onset and progressive course, even if it occurs after a stroke. Studies have shown that many patients hospitalized for stroke seems to have preexisting cognitive decline.

Dementia and neurocognitive disorders are important problems for health systems and a burden to society, with a significant impact as life expectancy increases. The autopsy and neuroimaging have shown that many cases of dementia are caused by pathological changes of vascular origin that occur in the brain (such as cerebral infarction, white matter lesions and microbleeds).

Dysfunction of the neurovascular unit and of the mechanisms that regulate cerebral blood flow are probably important components of the pathophysiological processes underlying VCI. Cerebral amyloid angiopathy appears as an important risk marker for Alzheimer's disease, microinfarction, microhemorrhage or macrohemorrhage of the brain and VCI. The pathogenesis of cognitive dysfunction in the elderly is generally a mixture of Alzheimer's disease and microvascular brain damage, which can cause, through overlap and synergy, an increased risk of cognitive impairment. In this regard, magnetic resonance imaging and other neuroimaging techniques may play an important role in the detection and definition of VCI, providing sufficient evidence that subcortical forms of VCI with white matter hyperintensities and small deep infarcts are common. (4)

Among patients who suffered a first stroke, post-stroke dementia (PSD) prevalence varies depending on the location, size of the infarction and the interval after the stroke. Generally, a stroke increases the risk of dementia by about 2 times. This is higher with 
s

advancing age, lower education level, history of diabetes or atrial fibrillation and recurrent cerebral infarction. Patients with PSD have impaired functionality in varying degrees and high mortality rates. (4) After adjusting the demographic factors, associated cardiac pathology, cerebral vascular event severity and stroke recurrence, the long-term mortality is two to six times higher in patients with post-stroke dementia.

\section{Risk factors for stroke and neurocognitive disorders}

Risk factors were associated with an increased incidence rate of the disease, higher chances of developing the illness or determining the early onset of the disease, depending on the type of statistical analysis performed. By comparison, the protective factors are the opposite. An observed risk factor does not necessarily cause the disease, just as a protective factor does not prevent the disease and certainly does not treat the disease.

In many studies, a higher number of cardiovascular risk factors in middle age, along with vascular disease and diabetes are associated with a higher risk of dementia and cognitive decline. An important means by which cardiovascular risk factors can cause cognitive dysfunction is by causing cerebrovascular events (such as stroke or TIA.

In the INTERSTROKE case-control study conducted between 11 January 2007 and 8 August 2015, on 26919 participants from 32 countries, it was shown that ten potentially modifiable risk factors are collectively associated with approximately $90 \%$ of the risks that can be attributable to the population (PAR - population attributable risks) for stroke, in every major region of the world, in ethnic groups, in women and men and in different age groups. (9)
History of blood pressure of $140 / 90 \mathrm{mmHg}$ or higher, physical inactivity, the ratio of apolipoprotein (Apo) B / ApoA1, diet, the waist / hip ratio, psychosocial factors, chronic tobacco use, cardiac causes, alcohol consumption and diabetes, have been associated with all types of stroke. Through an analysis that compared intracerebral hemorrhage with ischemic stroke (case-bycase analysis), hypertension was more strongly associated with cerebral hemorrhage, while smoking, diabetes, cardiac causes, and apolipoproteins were more significantly associated with ischemic stroke. (9)

Non-modifiable risk factors, also called risk markers, include demographic factors such as age, gender and race-ethnicity. In terms of age, generally, after the age of 65 there is an exponential increase in the incidence and prevalence of vascular origin dementia cases. Regarding sex, studies have shown a higher prevalence of stroke in women, this being justified by a higher life expectancy among them. Women are older at the onset of stroke compared to men (75 years compared to 71 years). Also, women between the ages of 4584 have a lower risk of stroke compared to men, but this association is reversed after the age of 85 , when women have a higher risk. (10) However, young women have a higher risk of stroke, which is correlated with pregnancy or postnatal status, hormonal changes and use of contraceptives.

In terms of race-ethnicity, studies have shown that black people have a double risk of stroke compared to their white counterparts, with a higher mortality associated with stroke. Latin Americans / Hispanics also have a higher risk. The disparity in the incidence of stroke is more prominent among young black people, in whom the risk of subarachnoid and intracerebral hemorrhage is substantially higher than whites of the same age. (11) 
Modifiable risk factors are divided into those of a medical nature that can be modified by appropriate treatment: hypertension, diabetes, atrial fibrillation and dyslipidemia, or lifestyle factors: diet, obesity, alcohol and / or tobacco consumption and physical activity.

\section{Modifiable medical risk factors}

High blood pressure (hypertension), defined as systolic blood pressure $\geq 140 \mathrm{mmHg}$ and diastolic blood pressure $\geq 90 \mathrm{mmHg}$, is the most important modifiable medical risk factor for both ischemic and hemorrhagic stroke. Maintaining blood pressure values within normal limits can significantly decrease the risk of cerebral vascular events, so that patients with BP $<120 / 80 \mathrm{mmHg}$ have about half the risk of people with hypertension.

Diabetes mellitus is an independent risk factor, increasing the risk of stroke by about two times, with $20 \%$ of diabetics dying by stroke. (11) Hyperglycaemia is associated with functional changes in cerebral circulation, but which are reversible when good glycemic control is restored. Studies have suggested that cognitive function is affected more as the presence of diabetes is longer. (4)

Atrial fibrillation (AF) is the most common cardiac arrhythmia, the most important complication of which is stroke of cardioembolic etiology. It is a strong risk factor for the ischemic stroke, especially when not properly treated with anticoagulant, increasing the risk of stroke by two to ten times among all age groups. (12)

The relationship between dyslipidemia and the risk of stroke is complex, with an increased risk of ischemic stroke associated with high levels of total cholesterol, but with a lower risk for high levels of HDLcholesterol. High cholesterol levels seems to be more strongly associated with ischemic stroke involving large arteries compared to other types of ischemic stroke. However, total cholesterol is inversely associated with hemorrhagic stroke, the risk increasing as total cholesterol decreases. According to the 2021 guideline for the prevention of stroke in patients with stroke and transient ischemic attack (a guideline from the American Heart Association/American Stroke Association) it is recommended that LDL-cholesterol levels be under $70 \mathrm{mg} / \mathrm{dl}$, to reduce cardiovascular events. (13)

\section{Modifiable risk factors associated with lifestyle}

Diet influences the risk of stroke, but also cardiovascular risk factors such as high blood pressure, diabetes and dyslipidemia. Salt intake, for example, is associated with a high risk of hypertension and stroke, while potassium intake is associated with a decreased risk of stroke ${ }^{(11)}$ Dietary guidelines for stroke prevention recommend reducing salt intake and increasing potassium intake, a diet rich in fruits and vegetables, or adopting DASH diet (Dietary Approaches to Stop Hypertension) or Mediterranean diet. (14)

Obesity increases the risk of stroke through multiple mechanisms, including hypertension, glucose intolerance, and changes in atherogenic serum lipids. Typically, body weight is divided into categories based on body mass index (BMI). (15) In recent years, abdominal obesity, as measured by the waist / hip ratio, proved to be a more important predictor of stroke. In the INTERSTROKE study, the body mass index had a lower association with stroke compared to the waist / hip ratio. (9)

Tobacco use remains a major risk factor for stroke, it doubles the risk compared to nonsmokers. Smoking is also associated with a 
two to four time's higher risk of subarachnoid hemorrhage (HSA). (15) Smoking cessation is an essential component in the prevention of stroke $^{(14)}$, with the risk of stroke being lower in people who have given up smoking compared to active smokers.

The effect of alcohol consumption on the risk of stroke is dependent on the amount of alcohol ingested and varies according to the type of stroke. Alcohol abuse can also affect other risk factors for stroke, leading to increased BP, higher rates of atrial fibrillation, coagulation disorders and reduced cerebral blood flow. However, moderate alcohol consumption can have beneficial effects by reducing platelet aggregation and plasma fibrinogen concentration, improving HDL-cholesterol and endothelial function. (15) There is evidence of a "J" form relationship between alcohol consumption and the risk of ischemic stroke, with low to moderate consumption having a protective effect against stroke, while abusive consumption is associated with an increased risk of ischemic stroke. (11) Current guidelines recommend moderate alcohol consumption, under two drinks per day for men and under one drink per day for nonpregnant women. (14)

Physical activity is the main subject of this article, being one of the factors that can bring significant benefits both on the risk of stroke, and on general health.

It is well known that physical inactivity or sedentary lifestyle is a predictor of cardiovascular disease and early mortality. Sedentary lifestyle is part of a vicious circle, and can easily cause obesity, dyslipidemia, atherosclerosis and arteriosclerosis, which will eventually lead to damage to all organs, all these changes causing difficulty or inability to perform physical activities. This vicious circle has a significant impact both on the quality of people's lives and on society and health systems, leading to a decrease in work capacity and an increase in the costs of preventing and treating acquired diseases.

The effects of physical activity are manifested by decreased blood pressure values, improvements in glucose tolerance, weight loss, reductions in fibrinogen and plasma platelet activity, increases in HDL-cholesterol and control of other cardiovascular risk factors. (15) At the cerebral level, physical activity increases neurotrophins, such as brain-derived neurotrophic factor, improves cerebrovascular function and cerebral perfusion, decreases the stress response and increases brain plasticity through synaptogenesis and neurogenesis. (4)

Physical activity is associated with a decreased risk of stroke and mortality, as well as favorable results after stroke, which makes it a potential preventive measure against cognitive decline. (16) The mechanism underlying these changes is not well known, but it seems to be related to changes in traditional risk factors, including the reduction of inflammation, oxidative stress and capillary dysfunction after stroke. $(17,18$, 19).

Although physical activity is recommended by both guidelines and international organizations in order to maintain physical and mental health, it is not so performed among the general population and stroke survivors. (20)

In the INTERSTROKE study conducted on 26,919 participants from 32 countries, physical activity decreased population attributable risk by about $53 \%$ in all types of stroke. (9) 


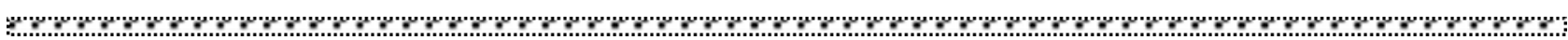

A combined program of aerobic and endurance exercises applied to patients with residual motor deficit post-stroke, significantly improved MoCA scores and reduced by $44.5 \%$ the number of patients who met the criteria for mild neurocognitive disorder. (21)

Although there are few data of the influence of physical activity performed before stroke, on cognitive function after stroke, this seems to have a significant importance. In the TALOS trial (The Efficacy of Citalopram Treatment in Acute Ischemic Stroke), there was a substudy performed on 488 patients. On them were applied the PASE scale (Physical Activity Scale for the Elderly) to assess the physical activity in the last seven days before the stroke and the scales SDMT (Symbol Digit Modalities Test) applied at one month and six months after stroke, respectively
MMSE (Mini -Mental State Examination) applied 6 months after stroke, to assess cognitive performance. This substudy demonstrated that an increased level of physical activity performed prior to stroke is independently associated with higher cognitive performance after stroke, measured by SDMT (16). This finding is consistent with previous findings that participation in physical activity and leisure is associated with better cognitive function after stroke (22).

The results obtained by these studies can assign to physical activity the role of protective factor. This makes it even more important to follow the stroke prevention guidelines, which recommend that healthy adults should perform moderate to intense physical activity for at least 40 minutes / day for 3-4 days a week. (14)

\section{CONCLUSIONS}

Conversion disorder has recently received attention in the psychiatric literature compared to other In the context of increasing life expectancy and survival after stroke, neurocognitive disorder is one of the main consequences of stroke, with a particular importance for the population in terms of quality of life and implicitly for society and health systems, in terms of costs.

Measures taken to reduce the risk factors can prevent neurocognitive disorders, as they reduce the frequency of developing cerebrovascular disease. The combined use of several measures with a vascular protective role, applied since middle age, could prevent several years of cognitive aging.

Regarding physical activity, there is ample evidence that it decreases the risk of stroke and the severity of complications that occur after it. However, studies on the relationship between physical activity performed before stroke, and cognitive performance after it, are relatively few, performed on small samples of participants but with encouraging results. This does not overshadow the benefit of physical activity on all cardiovascular risk factors and even offers the chance to conduct more extensive studies.

Therefore, according to international organizations, correlated with current medical guidelines, a healthy, balanced lifestyle is recommended in order to maintain biological constants within normal limits, which decreases the chances of developing cerebrovascular events that may impair cognitive function. However, in the event of a stroke, this lifestyle adopted both before and after the vascular event, can provide good functional results and improved cognitive performance during recovery.

\section{ACKNOWLEDGE AND DISCLOSURE}

The authors state that there are no declared conflicts of interest regarding this paper. 


\section{REFERENCES}

1. Vos T, Lim S, Abbafati C. Global burden of 369 diseases and injuries in 204 countries and territories, 1990-2019: a systematic analysis for the Global Burden of Disease Study 2019. The Lancet. 2020;1204-1222.

2. Sacco R, Kasner S, J B. An updated definition of stroke for the 21 st century: a statement for healthcare professionals from the American Heart Association/American Stroke Association. Stroke. 2013;2064-89.

3. American Psychiatric Association. Diagnostic and statistical manual of mental disorders: DSM-5 Washington, D.C.2013.

4. Gorelick P, Angelo S, Black S. Vascular contributions to cognitive impairment and dementia: a statement for healthcare professionals from the american heart association/american stroke association. Stroke. 2011; 2672-2713.

5. Lo JW, Crawford JD, Desmond DW. Profile of and risk factors for poststroke cognitive impairment in diverse ethnoregional groups. Neurology. 2019; 2257-2271.

6. Roman G. Vascular dementia prevention: A risk factor analysis. Cerebrovasc Dis. 2005;91-100.

7. Sachdev P, Lo WJ, Crawford J. STROKOG (stroke and cognition consortium): An international consortium to examine the epidemiology, diagnosis, and treatment of neurocognitive disorders in relation to cerebrovascular disease. Alzheimers Dementia. 2017; 1-23.

8. Offer A, Arnold M, Clarke R. Assessement of Vascular Event Prevention and Cognitive Function Among Older Adults With Preexisting Vascular Disease or Diabetes A Secondary Analysis of 3 Randomized Clinical Trials. Jama Network Open. 2019;1-2.

9. O'Donnell M, Chin S, Rangarajan S. Global and regional effects of potentially modifiable risk factors associated with acute stroke in 32 countries (INTERSTROKE): a case-control study. The Lancet. 2016;761-775.

10. Benjamin EJ, Blaha MJ, Chiuve SE. Heart Disease and Stroke Statistics-2017 Update: A Report From the American Heart Association. Circulation. 2017;146-603.

11. Boehme KA, Esenwa C, Elkind MSV. Stroke Risk Factors, Genetics, and Prevention. Circulation Research. 2017;472-495.

12. Kuklina EV, George MG, Bansil P. Epidemiology and prevention of stroke: a worldwide perspective. Expert Review of Neurotherapeutics. 2012;199-208.

13. Kleindorfer OD, Towfighi A, Chaturvedi S. Guideline for the Prevention of Stroke in Patients With Stroke and Transient Ischemic Attack: A Guideline From the American Heart Association/American Stroke Association. Stroke 2021.

14. Meschia JF, Bushnell C, Boden-Albala B. Guidelines for the primary prevention of stroke: a statement for healthcare professionals from the American Heart Association/American Stroke Association. Stroke. 2014; 37543832 .

15. Goldstein LB. Epidemiology of Cerebrovascular Disease. In Mark C. Vascular Medicine: A Companion to Braunwald's Heart Disease.: Elsevier; 2020;361-375.

16. Damsbo AG, Mortensen JK, Kraglund KL. Prestroke Physical Activity and Poststroke Cognitive Performance. Cerebrovascular Diseases. 2020;632-638.

17. Cancio EL, Ricciardi AC, Sobrino T. Reported Prestroke Physical Activity Is Associated with Vascular Endothelial Growth Factor Expression and Good Outcomes after Stroke. Journal of Stroke and Cerebrovascular Diseases. 2017; 425-430.

18. Hotting K, Roder B. Beneficial effects of physical exercise on neuroplasticity and cognition. Neuroscience \& Biobehavioral Reviews. 2013;2243-2257.

19. Hamakawa M, Ishida A, Tamakoshi K. Repeated short-term daily exercise ameliorates oxidative cerebral damage and the resultant motor dysfunction after transient ischemia in rats. Journal of Clinical Biochemistry and Nutrition. 2013;8-14.

20. Billinger AS, Arena R, Bernhardt J. Physical activity and exercise recommendations for stroke survivors: a statement for healthcare professionals from the American Heart Association/American Stroke Association. Stroke. 2014; 2532-2533. 
Bulletin of Integrative Psychiatry O New Series O June 2021 O Year XXVII ONo. 2 (89)/83

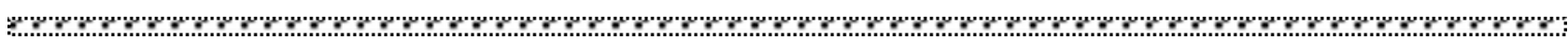

21. Marzolini S, Oh P, Mcllroy W. Neurorehabilitation and Neural Repair. Neurorehabilitation and Neural Repair. 2012;392-402.

22. Wong A, Lau AYL, Lo E. Relations between Recent Past Leisure Activities with Risks of Dementia and Cognitive Functions after Stroke. PLoS One. 2016.

23. Hankey G. Stroke. The Lancet. 2017;641-654.

\section{Correspondence:}

Roxana Chiriță,

M.D., PhD, Professor of Psychiatry University of Medicine and Pharmacy "Grigore T. Popa” Iași, Senior psychiatrist Socola Institute of Psychiatry, Iași, Romania, d.stigma@gmail.com

Submission: 02 may 2021

Acceptance: 08 jun 2021 